\section{Impfkampagnen: Nutzlose Aufklärung?}

In einer britisch-italienischen Studie wurden an 120 Studenten verschiedene Strategien getestet, um populäre Fehlurteile über Impfungen zu korrigieren. Zwei der drei Informationsstrategien führten zu einer deutlichen Verstärkung der Impfmythen. Die Ergebnisse zeigen nach Ansicht der Studienautoren, dass Fakten schnell aus dem Gedächtnis verschwinden und populäre Irrtümer zurückbleiben. Möglicherweise sei es sinnvoll, durch häufige und auf verschiedene Zielgruppen zugeschnittene Kampagnen die Verbreitung und Akzeptanz korrekten Impfwissens zu erhöhen.

Pluviano Set al. PLOS ONE 2017;12(7):e0181640

\section{Millionen dicke Kinder weltweit}

Die Zahl extrem dicker Kinder und Jugendlicher hat sich in den vergangenen vier Jahrzehnten mehr als verzehnfacht. 1975 waren weltweit etwa elf Millionen 5- bis 19-Jährige fettleibig , im vergangenen Jahr waren es 124 Millionen, berichten die Weltgesundheitsorganisation (WHO) und das Imperial College London zum Welt-Adipositas-Tag am 11. Oktober im Fachblatt "The Lancet". Dabei seien nur 10\% der Zunahme auf die wachsende Bevölkerungszahl zurückzuführen, berichtete Hauptautor Majid Ezzati vom Imperial College.

http://dx.doi.org/10.1016/S0140-6736(17)32129-3

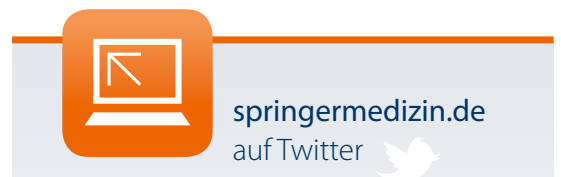

Auch im Herbst heißt es wieder:

Lauschen Sie unserem Zwitschern und werden Sie zum Follower.

- twitter.com/springermedizin

Urologischer Notfall

\title{
Penisrupturen häufig in der Hündchenstellung
}

Eine Penisruptur ereignet sich am ehesten bei der Hündchenstellung: Mehr als die Hälfte der Penisverletzungen beim Geschlechtsverkehr geschieht in dieser Position. Auf Platz zwei folgt die Missionarsstellung.

Urologen um Dr. Roberto Barros vom Souza Aguiar Municipal Hospital in Rio de Janeiro haben die Ursachen von Penisrupturen anhand einer Stichprobe von 90 Betroffenen untersucht. Drei Viertel der Patienten verletzten sich nach eigenen Angaben beim Geschlechtsverkehr, 16 hatten selbst Hand angelegt, nächtliches Überrollen war bei drei Männern der Auslöser, zwei erlitten ein stumpfes Trauma aufgrund anderer Ursachen.
Die 69 Männer mit Verletzungen während des Geschlechtsverkehrs wurden nach ihrer Position beim Koitus befragt. 54\% von ihnen nannten die Hündchenstellung (coitus a tergo), 33\% die Missionarsstellung, nur bei $13 \%$ befand sich die Frau oben.

Neun der Männer erlitten eine bilaterale Schwellkörperverletzung, sieben von ihnen während eines coitus a tergo. Danach sind die Verletzungen in dieser Position nicht nur häufiger, sondern oft auch gravierender. Die Frage nach der Position kann dem Operateur Auskunft darüber geben, ob er sich auf eine doppelseitige Fraktur der Corpora cavernosa oder eine Harnröhrenverletzung gefasst machen muss.

Barros Ret al. Int I Impot Res 2017; 29:207-9

\section{Ig-Nobelpreis für unwahrscheinliche Forschung}

\section{Warum sich viele Franzosen vor Käse ekeln}

Französische Spezialisten für funktionelle Magnetresonanztomografie (fMRT) haben den Ig-Nobelpreis 2017 für Medizin erhalten. Sie konnten zeigen, dass erstaunlich viele Franzosen Käse verabscheuen - und wo genau im Gehirn sich der Ekel manifestiert.

Fünf französische Forscher legten in ihrer Studie mithilfe der fMRT die neuronale Basis des Ekels gegenüber Käse offen. Dafür wurde ihnen der Ig-Nobelpreis 2017 für Medizin verliehen.

Den Resultaten der Studie mit 332 Französinnen und Franzosen folgend, liegt die Rate des Abscheus gegen Käse bei knapp 37\%. Käseverweigerer zeigten im fMRT höhere Aktivitäten im internen und externen Globus pallidus und in der Substantia nigra als Käse- liebhaber. Diese Hirngegenden spielen im Belohnungsverhalten eine Rolle, scheinen nach den Ergebnissen aber auch für aversives Verhalten von Bedeutung zu sein.

Die fünf französischen Forscher beschäftigen sich allgemein mit der Rolle des Geruchssinns bei Nahrungsmittelaversion, wie sie bei bestimmten Krankheiten vorkommt. Diese experimentell zu induzieren, halten sie für unethisch. So verfielen sie auf das Käsemodell, weil Käse die Menschen olfaktorisch und gustatorisch in klar abgegrenzte Lager scheidet.

Royet J et al. Front Hum 2016; 10: 511 